\title{
Plant miRNAs found in human circulating system provide evidences of cross kingdom RNAi
}

\author{
Yu-Chen Liu', Wen Liang Chen², Wei-Hsiang Kung ${ }^{1}$ and Hsien-Da Huang ${ }^{1,2,3,4^{*}}$ \\ From The Fifteenth Asia Pacific Bioinformatics Conference \\ Shenzhen, China. 16-18 January 2017
}

\begin{abstract}
Background: Emerging evidence indicates that plant miRNAs can present within human circulating system through dietary intake and regulate human gene expression. Hence we deduced that comestible plants miRNAs can be identified in the public available small RNA sequencing data sets.

Results: In this study, we identified abundant plant miRNAs sequences from 410 human plasma small RNA sequencing data sets. One particular plant miRNA miR2910, conserved in fruits and vegetables, was found to present in high relative amount in the plasma samples. This miRNA, with same 6mer and 7mer-A1 target seed sequences as hsa-miR-4259 and hsa-miR-4715-5p, was predicted to target human JAK-STAT signaling pathway gene SPRY4 and transcription regulation genes.

Conclusions: Through analysis of public available plasma small RNA sequencing data, we found the supporting evidence for the plant miRNAs cross kingdom RNAi within human circulating system.
\end{abstract}

Keywords: Circulating miRNAs, Plant miRNAs, miR2910, Cross Kingdom RNAi

\section{Background}

Micro RNAs (miRNA) are about 22 nucleotides single strand non-coding RNAs, which regulate protein coding genes expression through guiding protein-RNA complex toward mRNAs [1, 2]. Given the conventional recognition that expression of most of the genes are regulated by miRNAs in mammal, research of miRNAs' roles in pathogenesis in diseases and biological processes is foreseeable [3]. In recent years, stable cell free miRNAs had been found in human circulating system derived samples such as plasma, urine and saliva [4]. Circulating miRNAs are studied to be used as diagnosis biomarkers in many diseases [5].

Plant miRNA miR168a was found to present in the sera of mammals including human and regulate human

\footnotetext{
* Correspondence: bryan@mail.nctu.edu.tw

${ }^{1}$ Institute of Bioinformatics and Systems Biology, National Chiao Tung University, HsinChu, Taiwan

${ }^{2}$ Department of Biological Science and Technology, National Chiao Tung

University, HsinChu, Taiwan

Full list of author information is available at the end of the article
}

gene LDLRAP1 [6]. Positive and negative results of experimental test on whether exogenous miRNAs can present in animal circulating system through food ingestion has been published in the following years [7-10]. Until recently, another indicates that plant miRNAs can present within human circulating system through dietary intake and regulate human gene expression emerged. Broccoli sourced miR159 was found in human sera and proved to inhibit breast cancer growth through targeting the gene TCF7 [11].

Given the assumption that certain comestible plants miRNAs can be absorbed through ingestion and accumulate within human sera, we collected and examined 410 public available small RNA sequencing data sets, detected the existence of plant miRNAs through comparing to genomes of 5 different plant model organism Arabidopsis thaliana, Triticum aestivum, Oryza sativa, Zea mays, and Brachypodium distachyon. In results, we identified abundant plant miRNAs sequences from 410 human plasma small RNA sequencing data sets. Amount 
of these exogenous miRNA was compared to the ubiquitous human miRNA presented in the samples. One particular plant miRNA miR2910, conserved in fruits and vegetables, was found to present in high relative amount in the plasma samples. This miRNA, with same 6mer and 7mer-A1 target seed sequence as hsa-miR-4259 and hsamiR-4715-5p, was predicted to target human JAK-STAT signaling pathway gene SPRY4 and transcription regulation genes through miRTar [12].

This discovery not only provides new evidence of cross kingdom RNAi between human and plant, but also expanded the potential of dietary miRNA treatment on a wide range of variable diseases.

\section{Results and discussion}

A total 1,301 plant miRNAs, including 654 homologs, with at least one read presented in the sample were identified from the selected transcriptome sequencing runs. Count of the reads within each sample is available in (Additional file 1: Table S1). The relative abundance of these miRNAs were compared to detected human miRNAs. Among them we found a singular case, peuMIR2910, can be found ubiquitously within the selected samples. In some samples, up to more than one thousand copy of peu-MIR2910 can be found, which is more abundant than most of the human miRNAs detected within the samples. Beside peu-MIR2910, up to hundreds of copies of peu-MIR2916 in 379 samples, peu-MIR2914 in 359 samples, and tae-MIR2018 in 353 samples were also found.
The detected plant miRNAs are not human originated or sample contamination

Sequence alignment of the detected plant miRNAs sequence onto human genome was performed with BLAST [13] and resulted in zero alignment hit. Alignment of the conventional adaptor sequences to the plant miRNAs also resulted with zero hits. Through this result we are convinced that these detected plant miRNA sequence were not originated from conserved human sequences or adaptors contamination.

\section{The plant miRNA peu-MIR2910 is conserved within comestible plants}

Among the 1,301 plant miRNAs detected in our collected samples, miR2910 presented in all the 404 examined runs in relatively high abundance. Assuming that the source of plant miRNAs present in human plasma originated from food ingestion, then miR2910 must be evolutionally conserved in a wide variable kinds of comestible plants.

From the reports of recent years we found that peuMIR2910 was reported to be expressed within one of the model organism we used, Zea mays [14]. As one of the main grain consumed all over the world, maize can potentially the source of the miR2910 detected in the data sets. On the other hand, we also found that miR2910 is conserved within fruits and vegetables. Melon [15], Sorghum [16], tomato [17], tea [18] and oil palm [19] are also the potential source of miR2910. All these evidences support the assumption that the peu-MIR2910 present in the human plasma samples originate from food ingestion. On

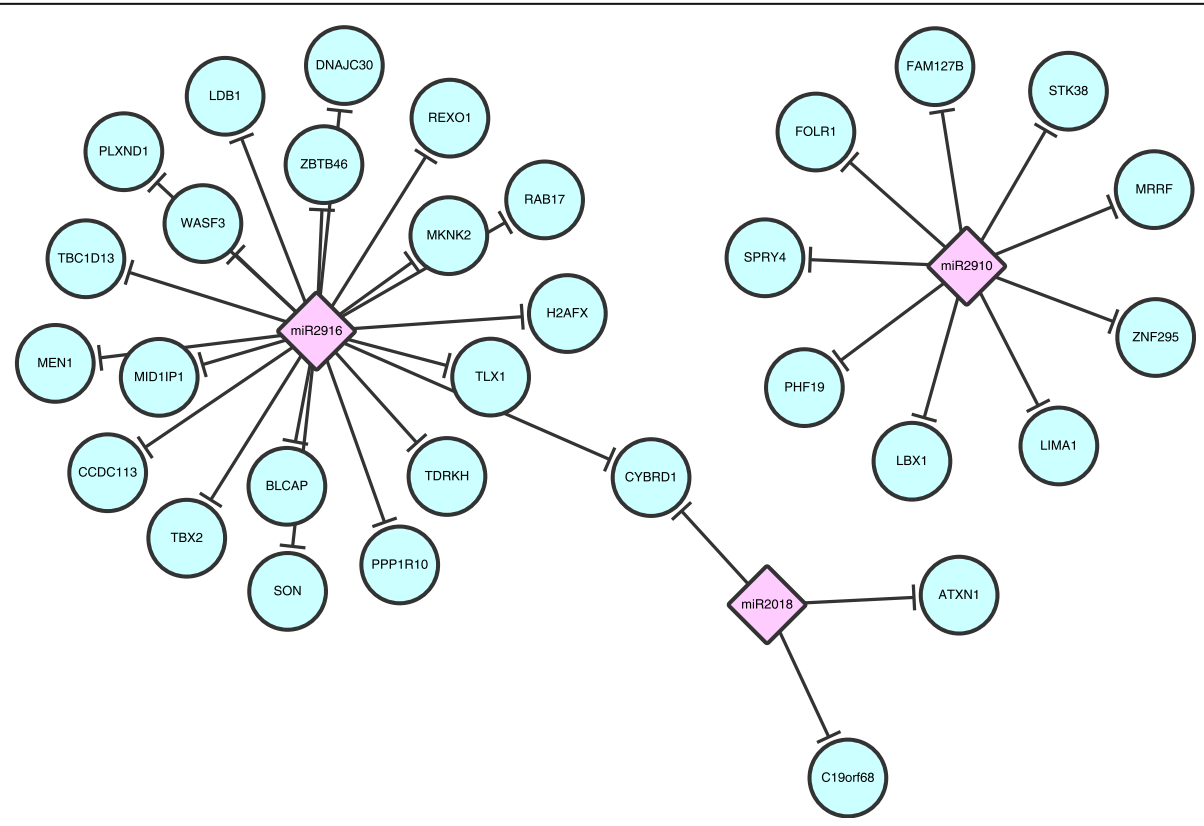

Fig. 1 Predicted target human genes of abundant plant miRNAs. The predicted miR2910, miR2916 and miR2018 targets through miRTar [12] are illustrated in this figure. The pink diamond shape nodes represent plant miRNAs while the round blue nodes represent human genes 
Table 1 Gene set enrichment of miR2910 target genes

\begin{tabular}{llll}
\hline Category & Term & PValue & Genes \\
\hline GOTERM_CC_FAT & GO:0042995 cell projection & 0.026595 & FOLR1, CTNND1, SPRY4 \\
SP_PIR_KEYWORDS & developmental protein & 0.048812 & LBX1, STK36, SPRY4 \\
GOTERM_BP_FAT & GO:0045449 regulation of transcription & 0.049408 & LBX1, PHF19, STK36, ZNF295, CTNND1 \\
INTERPRO & IPR011989: Armadillo-like helical & 0.059853 & STK36, CTNND1 \\
SP_PIR_KEYWORDS & transcription regulation & 0.060318 & LBX1, PHF19, ZNF295, CTNND1 \\
SP_PIR_KEYWORDS & Transcription & 0.06372 & LBX1, PHF19, ZNF295, CTNND1 \\
\hline
\end{tabular}

This table summarized the gene set enrichment of miR2910 targets. The human genes listed here were predicted to be targeted by plant miR2910 through sequence analysis. The terms were originated from the web based tool DAVID [33]

the other hand, peu-MIR2914 and peu-MIR2916 were also found conserved within oil palm [20]. Conserved taeMIR2018 was found in wheat $[21,22]$.

\section{Plant miR159 and miR168a were also detected in the examined samples}

To verify the analysis result with previous reports, we examined the amount of detected miR159 and miR168a within the examined samples. As listed in the (Additional file 1: Table S1), despite presenting in relatively small amount (less than 4 copies), miR159 can be found in 32 samples while miR168a can be found in 7 samples. The difference might be result from the difference of the diet habit. Most of the examined samples were presumably collected from Wisconsin, USA [23]. The dietary habit of the subjects can be much different from the subjects collect in China [6] and California [11]. On the other hand, given the fact that only roughly $1 \%$ of all the adaptor trimmed reads can be aligned onto plant genomes, as well as the generally low concentration of miRNA within plasma samples, detection of the miR159 as well as miR168a in less than 4 copy within one sample can still be considered as supporting evidence of the two previous reports.

\section{Human genes can potentially be targeted by peu- MIR2910 and other plant miRNAs}

In the previous reports $[6,11]$, plant miRNAs present in human circulating system were proven to be able to target human genes through similar AGO protein correlated mechanism. Given the assumption that miRNAs with similar or same target seed sequences can target

Table 2 Gene set enrichment of miR2916 target genes

\begin{tabular}{llll}
\hline Category & Term & P Value & Genes \\
\hline KEGG_PATHWAY & ptr05202: Transcriptional misregulation in cancer & 0.011556 & MEN1, LDB1, TLX1 \\
GOTERM_BP_DIRECT & GO:0043484 regulation of RNA splicing & 0.02339 & SON \\
INTERPRO & IPR000467:G-patch domain & 0.025805 & SON \\
UP_KEYWORDS & DNA-binding & 0.028196 & TBX2, PPP1R10, H2AFX, TLX1 \\
INTERPRO & IPR014720: Double-stranded RNA-binding-like domain & 0.029124 & SON \\
GOTERM_CC_DIRECT & GO:0071011 precatalytic spliceosome & 0.033471 & SON \\
SMART & SM00443:G_patch & 0.036062 & SON \\
GOTERM_BP_DIRECT & GO:0000910 cytokinesis & 0.036161 & SON \\
GOTERM_MF_DIRECT & GO:0003723 RNA binding & 0.037823 & SON, TDRKH \\
GOTERM_CC_DIRECT & GO:0000781 chromosome, telomeric region & 0.046328 & PPP1R10, H2AFX \\
GOTERM_BP_DIRECT & GO:0006397 mRNA processing & 0.053776 & SON \\
GOTERM_BP_DIRECT & GO:0000226 microtubule cytoskeleton organization & 0.058753 & SON \\
GOTERM_BP_DIRECT & GO:0032092 positive regulation of protein binding & 0.063706 & MEN1, PLXND1 \\
GOTERM_CC_DIRECT & GO:0000785 chromatin & 0.073822 & MEN1, PPP1R10 \\
UP_KEYWORDS & Nucleus & 0.081889 & TBX2, PPP1R10, H2AFX, TLX1 \\
GOTERM_CC_DIRECT & GO:0005634 nucleus & 0.08342 & MEN1, TBX2, LDB1, MKNK2, H2AFX, MID1IP1, TLX1 \\
GOTERM_BP_DIRECT & GO:0051726 regulation of cell cycle & 0.091709 & SON \\
\hline
\end{tabular}

This table summarized the gene set enrichment of miR2916 targets. The human genes listed here were predicted to be targeted by plant miR2916 through sequence analysis. The terms were originated from the web based tool DAVID [33] 
same sets of genes, we tested the similarity of plant miRNAs and human miRNAs in target seed regions.

Through the result of the target seed analysis, we found that despite the whole sequence, including the hairpin, of peu-MIR2910 cannot be aligned onto human genome, the 6mer and 7mer-A1 target seed sequences of miR2910, CCAACT and CCAACTA, is the same as hsa-miR-4259 and hsa-miR-4715-5p. Given the previously reported case of miR168a [6] and miR159 [11], we hypothesis that peuMIR2910 within the circulating system can target human genes as well. From the 51 target genes of hsa-miR-4259 and hsa-miR-4715-5p reported in miRTarBase [24], and the mature sequence of miR2910, we found 10 possible target genes predicted through miRTar [12], as illustrated in Fig. 1. We found that miR2910 can potentially target on 5' UTR of LIMA1, and the CDS of CTNND1, FOLR1, LBX1 STK38, FAM127B, PHF19, ZNF295, SPRY4 and MRRF.

Interestingly, we also found that miR2916 has exact same 8mer target seed sequence, AGTCCCCA, with human miRNA hsa-miR-4652-5p. From the 40 target genes of hsa-miR-4652-5p reported in miRTarBase [24], and the mature sequence of miR2916, we found other 20 possible target genes predicted through miRTar [12]. Same analysis process was conducted on miR2018, and three potential human gene targets were found. These findings were also illustrated in Fig. 1.

From the gene sets enrichment, we found that LBX1, PHF19, STK36, ZNF295 and CTNND1 are associated with regulation of transcription, while SPRY 4 belongs to human JAK-STAT signaling pathway, as summarized in Table 1. CTNND1 is also associated with Adherens

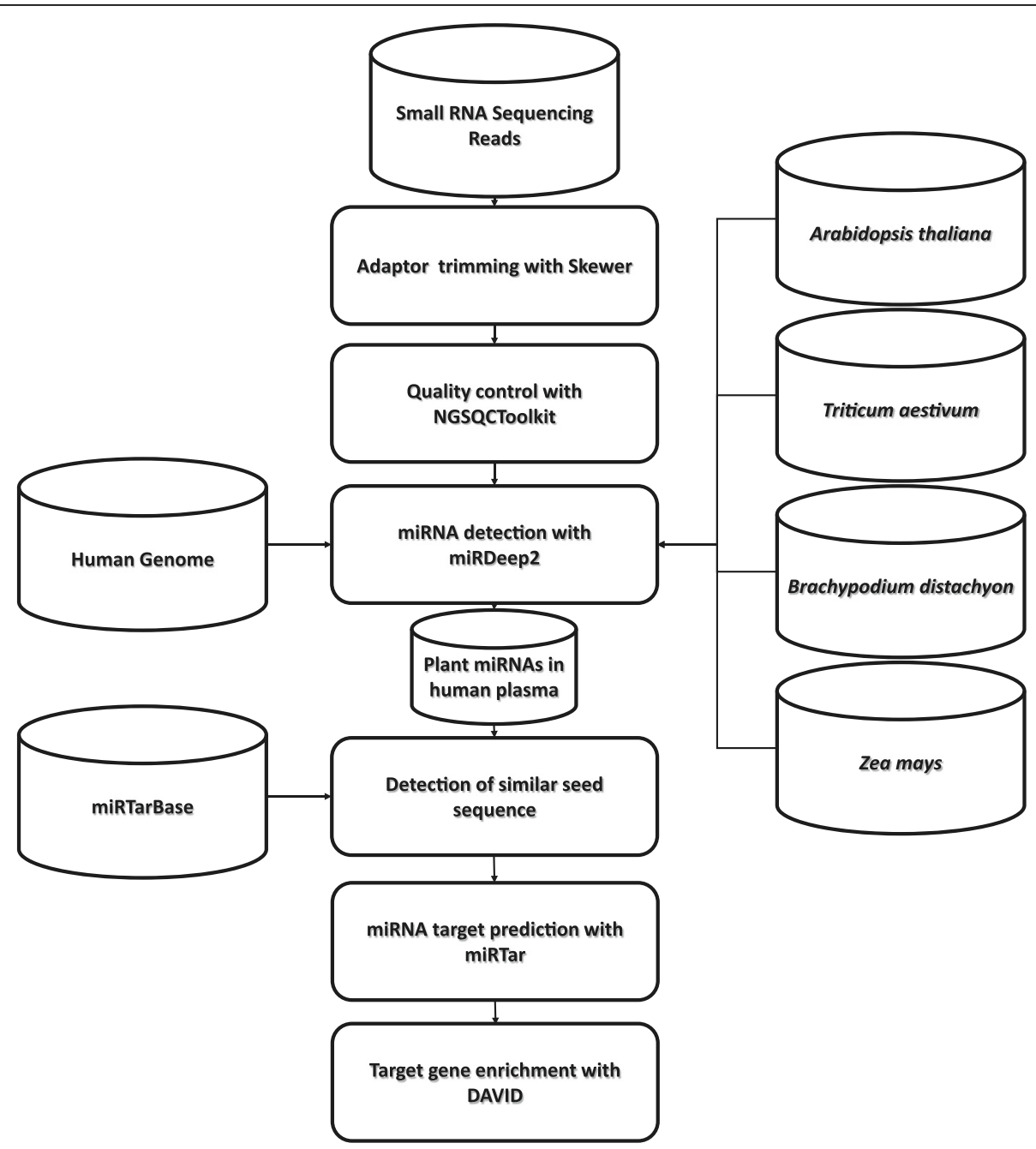

Fig. 2 Summary of the data analysis process. The data analysis pipeline designed to detect the comestible plants miRNAs in human plasma samples is summarized in this figure. The collected human circulating small RNA-seq reads were align to the plant genome. The existence of plant miRNAs was examined through the algorithm of miRDeep2 package [29] 
junctions and endocytosis [25]. We also found that three genes MEN1, LDB1 and TLX1, predicted to be targeted by miR2916 were associated with Transcriptional misregulation in cancer, as summarized in Table 2.

\section{Methods}

The data analysis process in this research is summarized in Fig. 2. Small RNA sequencing data sets of human plasma was dowloaded from NCBI SRA [26]. The adaptors within the data set were trimmed with the tool Skewer [27]. Quality control of the remaining reads was further conducted through NGSQCToolkit [28]. The miRNA detection was performed through miRDeep 2 [29]. To detect the plant miRNAs within the samples, we collected the genomes of 5 different plant model organism Arabidopsis thaliana, Triticum aestivum, Oryza sativa, Zea mays, and Brachypodium distachyon. The assembly version used were GCA_000001735.1, GCA_000005505.2, GCA_001305255.1, GCA_000210335.1 and GCA_00021 0335.1, which were downloaded from NCBI assembly. The plant miRNAs used for detection were collected from the database PMRD [30].

The human plasma small RNA sequencing reads were aligned onto each of the plant genomes through Bowtie [31] and the miRDeep2 package [29]. Whether the plant miRNAs presented in the sample was determined by the copy of read aligned on the genomes, precursor and mature sequences of miRNAs. On the other hand, the HG19 human genome was used to detect the human miRNA. The target seed sequences [3] of the detected plant miRNAs were compared to the human mature miRNA sequence downloaded from miRBase [32]. Target genes of human miRNAs with close target seed sequences were assumed to be potentially targeted by the plant miRNAs. The target gene of these human miRNAs were acquired from miRTarBase [24]. Finally these target genes and the sequence of the interested plants miRNAs were used as candidates for miRNA target prediction. The target prediction was conducted through miRTar [12]. The target gene prediction of miRNAs was conducted on 3'UTR, CDS and 5'UTR of the genes, with the threshold of MFE $\leqq-14$ and score $\geqq 140$. The gene set enrichment was performed with DAVID [33].

\section{Conclusions}

Through analysis of public available plasma small RNA sequencing data, we found the supporting evidence for the plant miRNAs cross kingdom RNAi within human circulating system.

Singular amount of plant miRNA peu-MIR2910, conserved in fruits and vegetables, was found in the plasma samples presumably collected from the population of Wisconsin, USA. This miRNA, with same 6 mer and
7mer-A1 target seed sequences as hsa-miR-4259 and hsa-miR-4715-5p, CCAACT and CCAACTA, was predicted to target human JAK-STAT signaling pathway gene SPRY4 and transcription regulation genes. Whether the detected miR2910 can be absorbed into circulating system through food ingestion should be further tested through xenograft experiments.

\section{Additional file}

Additional file 1: contains supplemental table 1, which summarize count of the reads of plant miRNAs found within each sample. (CSV 2 mb)

\section{Acknowledgements \\ The authors would like to thank the Ministry of Science and Technology and the National Chiao Tung University and Ministry of Education, Taiwan, R.O.C. Veterans General Hospitals and University System of Taiwan (VGHUST), for financially supporting this research.}

\section{About this supplement}

This article has been published as part of BMC Genomics Volume 18 Supplement 2, 2017. Selected articles from the 15th Asia Pacific Bioinformatics Conference (APBC 2017): genomics. The full contents of the supplement are available online http://bmcgenomics.biomedcentral.com/articles/supplements/ volume-18-supplement-2

\section{Funding}

This work is supported by multiple programs from the Ministry of Science and Technology, Taiwan [MOST 105-2627-M-009-007-, MOST 103-2628-B-009-001-MY3, MOST 105-2319-B-400-002-, MOST 104-2911-I-009-509, and MOST 105-2633-B-009003-, MOST 104-2314-B-195-014]. This study was also supported by research grants from Health and welfare surcharge of tobacco products, Ministry of Health and Welfare, Taiwan [MOHW105-TDU-B-212-134002]. Finally, this work is particularly supported by "Aiming for the Top University Program" of the National Chiao Tung University and Ministry of Education, Taiwan, R.O.C. Veterans General Hospitals and University System of Taiwan (VGHUST) Joint Research Program IVGHUST105-G14-2]. The funding includes the cost of labor force, publication costs of the manuscript and maintenance fee of the research facilities as well as the computational devices.

Availability of data and materials

Supplemental table 1 is available in the Additional file 1.

\section{Authors' contributions}

Y.C. L. proposed and conducted this study. H.D.H. supervised the progress of the study, provided guidance in its design and coordination. W.L.C, W.H. K. and H.D.H. helped to draft and revise the manuscript. All read and approved the final manuscript.

Competing interests

The authors declare that they have no competing interests.

Consent for publication

Not applicable.

Ethics approval and consent to participate Not applicable.

\section{Author details}

${ }^{1}$ Institute of Bioinformatics and Systems Biology, National Chiao Tung University, HsinChu, Taiwan. ${ }^{2}$ Department of Biological Science and Technology, National Chiao Tung University, HsinChu, Taiwan. ${ }^{3}$ Center for Bioinformatics Research, National Chiao Tung University, Hsinchu 300, Taiwan. ${ }^{4}$ Department of Biomedical Science and Environmental Biology, Kaohsiung Medical University, Kaohsiung 300, Taiwan. 


\section{Published: 14 March 2017}

\section{References}

1. Pradère U, Hall J. Site-Specific Difunctionalization of Structured RNAs Yields Probes for microRNA Maturation. Bioconjug Chem. 2016;27(3):681-7.

2. Cai $Y, Y u X, H u S, Y u J$. A brief review on the mechanisms of miRNA regulation. Genomics Proteomics Bioinformatics. 2009;7(4):147-54.

3. Bartel DP. MicroRNAs: genomics, biogenesis, mechanism, and function. Cell. 2004:116(2):281-97.

4. Kosaka N, Iguchi H, Ochiya T. Circulating microRNA in body fluid: a new potential biomarker for cancer diagnosis and prognosis. Cancer Sci. 2010;101(10):2087-92.

5. Schwarzenbach $H$, Hoon DS, Pantel K. Cell-free nucleic acids as biomarkers in cancer patients. Nat Rev Cancer. 2011;11(6):426-37.

6. Zhang L, Hou D, Chen X, Li D, Zhu L, Zhang Y, Li J, Bian Z, Liang X, Cai $X$, et al. Exogenous plant MIR168a specifically targets mammalian LDLRAP1: evidence of cross-kingdom regulation by microRNA. Cell Res. 2012;22(1):107-26

7. Denzler R, Stoffel M. Uptake and function studies of maternal milk-derived microRNAs. J Biol Chem. 2015;290(39):23680-91.

8. Dickinson B, Zhang Y, Petrick JS, Heck G, Ivashuta S, Marshall WS. Lack of detectable oral bioavailability of plant microRNAs after feeding in mice. Nat Biotechnol. 2013:31(11):965-7.

9. Micó V, Martín R, Lasunción MA, Ordovás JM, Daimiel L. Unsuccessful Detection of Plant MicroRNAs in Beer, Extra Virgin Olive Oil and Human Plasma after an Acute Ingestion of Extra Virgin Olive Oil. Plant Foods Hum Nutr. 2016;71(1):102-8.

10. Baier SR, Nguyen C, Xie F, Wood JR, Zempleni J. MicroRNAs are absorbed in biologically meaningful amounts from nutritionally relevant doses of cow milk and affect gene expression in peripheral blood mononuclear cells, HEK-293 kidney cell cultures, and mouse livers. J Nutr. 2014;144(10):1495-500.

11. Chin AR, Fong MY, Somlo G, Wu J, Swiderski P, Wu X, Wang SE. Cross-kingdom inhibition of breast cancer growth by plant miR159. Cell Res. 2016;26(2):217-28.

12. Hsu JB-K, Chiu C-M, Hsu S-D, Huang W-Y, Chien C-H, Lee T-Y, Huang H-D. miRTar: an integrated system for identifying miRNA-target interactions in human. BMC bioinf. 2011;12:1

13. Altschul SF, Madden TL, Schäffer AA, Zhang J, Zhang Z, Miller W, Lipman DJ. Gapped BLAST and PSI-BLAST: a new generation of protein database search programs. Nucleic Acids Res. 1997;25(17):3389-402.

14. Wang Y-G, An M, Zhou S-F, She Y-H, Li W-C, Fu F-L. Expression profile of maize microRNAs corresponding to their target genes under drought stress. Biochem Genet. 2014;52(11-12):474-93

15. Gonzalez-Ibeas D, Blanca J, Donaire L, Saladié M, Mascarell-Creus A, CanoDelgado A, Garcia-Mas J, Llave C, Aranda MA. Analysis of the melon (Cucumis melo) small RNAome by high-throughput pyrosequencing. BMC Genomics. 2011;12(1):1.

16. Zhang L, Zheng Y, Jagadeeswaran G, Li Y, Gowdu K, Sunkar R. Identification and temporal expression analysis of conserved and novel microRNAs in Sorghum. Genomics. 2011;98(6):460-8.

17. Sablok G, Luo C, Lee WS, Rahman F, Tatarinova TV, Harikrishna JA, Luo Z. Bioinformatic analysis of fruit-specific expressed sequence tag libraries of Diospyros kaki Thunb: view at the transcriptome at different developmental stages. 3 Biotech. 2011;1:35-45.

18. Das A, Mondal TK. Computational identification of conserved microRNAs and their targets in tea (Camellia sinensis). Am J Plant Sci. 2010;1(02):77.

19. Low E-TL, Rosli R, Jayanthi N, Azizi N, Chan K-L, Maqbool NJ, Maclean P, Brauning R, McCulloch A, Moraga R. Analyses of hypomethylated oil palm gene space. PLoS ONE. 2014;9(1), e86728.

20. Singh R, Ong-Abdullah M, Low E-TL, Manaf MAA, Rosli R, Nookiah R, Ooi LC-L, Ooi SE, Chan K-L, Halim MA. Oil palm genome sequence reveals divergence of interfertile species in Old and New worlds. Nature. 2013;500(7462):335-9.

21. Wei B, Cai T, Zhang R, Li A, Huo N, Li S, Gu YQ, Vogel J, Jia J, Qi Y. Novel microRNAs uncovered by deep sequencing of small RNA transcriptomes in bread wheat (Triticum aestivum L.) and Brachypodium distachyon (L.) Beauv. Funct Integr Genomics. 2009;9(4):499-511.

22. Lucas SJ, Budak H. Sorting the wheat from the chaff: identifying miRNAs in genomic survey sequences of Triticum aestivum chromosome 1AL. PLoS ONE. 2012:7(7), e40859.

23. Yuan T, Huang X, Woodcock M, Du M, Dittmar R, Wang Y, Tsai S, Kohli M, Boardman L, Patel T. Plasma extracellular RNA profiles in healthy and cancer patients. Sci Rep. 2016;6:23202.

24. Chou C-H, Chang N-W, Shrestha S, Hsu S-D, Lin Y-L, Lee W-H, Yang C-D, Hong H-C, Wei T-Y, Tu S-J. miRTarBase 2016: updates to the experimentally validated miRNA-target interactions database. Nucleic Acids Res. 2016; 44(D1):D239-47.

25. Kanehisa M, Goto S. KEGG: kyoto encyclopedia of genes and genomes. Nucleic Acids Res. 2000;28(1):27-30.

26. Leinonen $R$, Sugawara $H$, Shumway M. The sequence read archive. Nucleic Acids Res. 2010;39:D19-21.

27. Jiang H, Lei R, Ding S-W, Zhu S. Skewer: a fast and accurate adapter trimmer for next-generation sequencing paired-end reads. BMC bioinf. 2014;15(1):1.

28. Patel RK, Jain M. NGS QC Toolkit: a toolkit for quality control of next generation sequencing data. PLoS ONE. 2012;7(2), e30619.

29. Friedländer MR, Mackowiak SD, Li N, Chen W, Rajewsky N. miRDeep2 accurately identifies known and hundreds of novel microRNA genes in seven animal clades. Nucleic Acids Res. 2012:40(1):37-52

30. Zhang Z, Yu J, Li D, Zhang Z, Liu F, Zhou X, Wang T, Ling Y, Su Z. PMRD: plant microRNA database. Nucleic Acids Res. 2010;38 suppl 1:D806-13.

31. Kinkhabwala A, Yu Z, Fan S, Avlasevich Y, Müllen K, Moerner W. Large single-molecule fluorescence enhancements produced by a bowtie nanoantenna. Nat Photonics. 2009:3(11):654-7.

32. Kozomara A, Griffiths-Jones S. miRBase: annotating high confidence microRNAs using deep sequencing data. Nucleic Acids Res. 2014;42(D1):D68-73.

33. Huang DW, Sherman BT, Tan Q, Collins JR, Alvord WG, Roayaei J, Stephens R, Baseler MW, Lane HC, Lempicki RA. The DAVID Gene Functional Classification Tool: a novel biological module-centric algorithm to functionally analyze large gene lists. Genome Biol. 2007:8(9):1.

\section{Submit your next manuscript to BioMed Central and we will help you at every step:}

- We accept pre-submission inquiries

- Our selector tool helps you to find the most relevant journal

- We provide round the clock customer support

- Convenient online submission

- Thorough peer review

- Inclusion in PubMed and all major indexing services

- Maximum visibility for your research

Submit your manuscript at www.biomedcentral.com/submit
) Biomed Central 